\title{
Quantitative Assessment of Cysteine and Cystine in Peptides and Proteins Following Organomercurial Derivatization and Analysis by Matrix-Assisted Laser Desorption Ionization Mass Spectrometry
}

\author{
Eugene J. Zaluzec, Douglas A. Gage, and J. Throck Watson \\ Department of Biochemistry, Michigan State University, East Lansing, Michigan, USA
}

\begin{abstract}
Protocols for the analysis of the sulfhydryl content in peptides and proteins using chemical derivatization by organomercurial reagents and analysis by matrix-assisted laser desorption ionization mass spectrometry (MALDI-MS) have been developed. The number of reactive cysteine residues in peptides and proteins can be determined by exploiting the affinity and selectivity of organomercurial reagents for macromolecular thiols. Mass shifts observed in MALDI mass spectra obtained before and after cysteine derivatization with p-hydroxymercuribenzoate ( $\mathrm{pHMB}$ ) permit the number of free sulfhydryl groups to be determined. The pHMB derivative of each free cysteine residue provides a mass shift of $321 \mathrm{u}$, overcoming limitations in the mass resolution of MALDI time-of-flight mass spectrometry. Reactive cysteine residues in a macromolecule can be selectively derivatized by using a fivefold molar excess of pHMB reagent. Total sulfhydryl content (i.e., cysteine and cystine) can be determined after disulfide reduction. However, analyses for total cysteine content are more complex, requiring protein denaturation, cystine reduction, and sample purification before derivatization and analysis by MALDI-MS. Conditions for sample denaturation, alkyl-phosphine reduction, $\mathrm{pHMB}$ derivatization, and sample purification by analyte adsorption and desalting on protein transfer membranes, are described for cysteine/cystine analysis performed on microgram (10-200 pmol) quantities of somatostatin, insulin, hemoglobin, and $\beta$-lactoglobulin. (J Am Soc Mass Spectrom 1994, 5, 359-366)
\end{abstract}

$\mathrm{N}$ umerous methods for the quantitative determination of free sulfhydryl groups (cysteine) and disulfide-bonds (cystine) in proteins are based on selective reactions in which thiols participate. The most precise and commonly used methods involve spectrophotometric titrations with mercaptide-forming compounds $[1,2]$ or alkylating reagents $[3,4]$, while others incorporate amino acid analysis following conversion of cysteine to cysteic acid [5] or to an alkyl derivative $[4,6,7]$. Major limitations in the spectrophotometric titration of macromolecular thiols are the relatively large sample size requirement (in the milligram range) and the uncertainty in determination of the end point. Although amino acid analyses can be performed at the microgram level, differentiating between free cysteine residues and those involved in disulfide bonds (cystines) can be cumbersome, as this involves first modifying the initially free sulfhydryl groups, remov-

Address reprint requests to Dr. J. Throck Watson, Department of Biochemistry, Michigan State University, East Lansing, MI 48824. ing the excess reagents, reducing the disulfide bonds, then modifying the nascent sulfhydryl groups in a way that allows for their recognition [8, 9]. Furthermore, because amino acid analysis provides information on the total amino acid content of the sample, a protein contamination of only $10 \%$ could lead to an error of $10 \%$ or more in the quantitation of derivatized cysteine residues.

Mass spectral analysis of derivatized macromolecular thiols is an alternative means of quantitatively assessing protein cysteine/cystine content in a way that promises to overcome limitations of sensitivity, multiple derivatizations, and protein contamination that burden the more classical methods. Because heterogeneous protein samples can be analyzed at the picomole level by matrix-assisted laser desorption ionization mass spectrometry (MALDI-MS) [10, 11], analysis of the mass spectral shifts associated with selective chemical derivatization of the analyte has the potential to become a sensitive means for analyzing the sulfhydryl content of peptides and proteins. 
One means to selectively derivatize macromolecular thiols exploits the reactivity they display toward metal ions. Although many monovalent and divalent metal ions can react with sulfhydryl groups, mercurial reagents are known to be especially selective for thiols because the strong mercury-sulfur affinity greatly exceeds the interactions that may occur between mercury and other types of reactive groups typically present in a protein [12]. However, divalent mercuric ions $\left(\mathrm{Hg}^{{ }^{+}+}\right)$ are capable of reacting in a way to "bridge" two thiol groups [13], and also have the potential of reacting with existing disulfide bonds. In contrast, monofunctional organomercurial compounds of the type $\mathrm{RHgX}$ have the advantage of only reacting with one thiol group. A commonly used compound in this class (Figure 1a) is p-hydroxymercuribenzoate (pHMB), which has been used in the spectrophotometric and amperometric determination of sulfhydryl groups in proteins $[14,15]$.

We report here a new method of determining the number of cysteine and cystine residues present in peptides and proteins using chemical derivatization by a monofunctional organomercurial reagent prior to analysis by MALDI-MS. In these analyses, a peptide or denatured protein is first allowed to react with a monovalent organic mercurial reagent in the absence

a

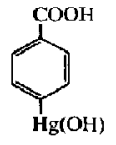

p-(Hydroxymercuri)-

benzoic acid

mass shift $=321$<smiles>O=S(=O)(O)c1ccc(CO)cc1</smiles>

p-(Hydroxymercuriphenyl)sulfonic acid

mass shift $=357$

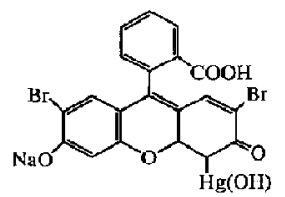

Merbromin

mass shift $=764.8$ b

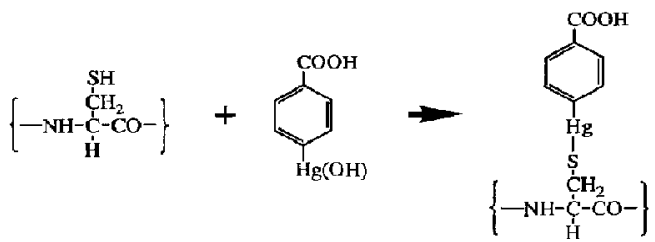

Figure 1. (a) Structures of organomercurials used for derivatization of sulfhydryl groups in proteins prior to analysis by MALDIMS. (b) According to the reaction scheme, derivatization of each sulfhydryl group causes a mass shift of $321 \mathrm{u}$ for pHMB (and 357 $u$ for pHMBS and $690 \mathrm{u}$ for merbromin). of a disulfide reducing agent to selectively label any free sulfhydryl group(s). Parallel experiments using underivatized protein provide mass spectral data from which to calculate the number of free sulfhydryls by the shift in the molecular weight of the organomercurial/protein derivative relative to that of the native protein. In the case for pHMB, a mass shift of $321 \mathrm{u}$ units is observed for each derivatized cysteine of the protein (Figure 1b). A complementary experiment involving the same reaction, after exposure of the sample to a disulfide reducing reagent, provides mass spectral data that allow for differentiation of the total number of cysteine(s) and/or cystine(s) originally present. Conditions for protein denaturation, cystine reduction with alkyl-phosphine reagents, pHMB derivatization, and sample purification by analyte adsorption on protein transfer membranes following removal of excess reagent by aqueous washing are described for the selective and sensitive determination of cysteine/cystine residues in proteins on microgram quantities of sample.

\section{Experimental}

MALDI mass spectra were obtained on a Vestec LaserTec Research (Vestec Corp., Houston, TX) timeof-flight (TOF) mass spectrometer equipped with a nitrogen laser (337nm, 3-ns pulse). The accelerating voltage in the ion source was set to $28 \mathrm{kV}$. Data were acquired in the linear mode of operation, using a 500-Mhz transient recorder with 1-ns resolution. Each spectrum was produced by accumulating data collected from 64 laser shots. Time-to-mass conversion was achieved by internal calibration using standards of bradykinin, oxidized insulin B chain, or insulin (5 pmol of each standard) obtained from Sigma Chemical Co. (St. Louis, MO).

Derivatization was performed with p-hydroxymercuribenzoate ( $\mathrm{pHMB}$ Sigma), p-hydroxymercuriphenylsulfonate ( $\mathrm{pHMPS}$, Sigma), or merbromin (Fluka Chemie AG, Buchs, Switzerland). Approximately 500 $\mathrm{mg}$ of pHMB reagent were washed with $1.0 \mathrm{~mL}$ of an aqueous $0.1 \%$ trifluoroacetic (TFA) acid solution three times to remove contaminating mercuric $\left(\mathrm{Hg}^{++}\right)$salts, filtered, and dried before using. Solutions of the pHMB $(2.8 \mathrm{mg} / \mathrm{mL})$ were prepared freshly in either $0.1 \%$ $\mathrm{NH}_{4} \mathrm{OII}$ or $0.1 \% \mathrm{NH}_{4} \mathrm{OH}$ made $4 \mathrm{M}$ in urea and vortexed until dissolved. Merbromin and $\mathrm{pHMPS}$ were used as supplied. Hemoglobin samples were supplied by Dr. T. Yang, Department of Chemistry, University of Wisconsin-Eau Clare, Eau Clare, Wisconsin. All other peptides and proteins were purchased from Sigma. Peptide and protein samples (1.0 $\mu \mathrm{g}$ unless otherwise stated) were solubilized in $5.0 \mu \mathrm{L}$ of either $0.1 \%$ $\mathrm{NH}_{4} \mathrm{OH}$ or $0.1 \% \mathrm{NH}_{4} \mathrm{OH}$ made $4 \mathrm{M}$ in urea as described in the text. Reduction of cystine was performed with a solution of tris(2-carboxyethyl)phosphine hydrochloride (TCEP, purchased from Pierce, Rockford, IL) prepared in $0.1 \% \mathrm{NH}_{4} \mathrm{OH}$ to provide a twofold 
molar excess of the reducing reagent over cystine in a water bath at $37{ }^{\circ} \mathrm{C}$ for 20 minutes. Derivatization of the free sulfhydryl groups was achieved by adding a fivefold molar excess of the organomercurial reagent (over free cysteine) and reacting for 5-20 minutes at room temperature. Samples containing urea were desalted by diluting twentyfold with a 1:1 solution of acetonitrile/aqueous $0.1 \%$ TFA, then applying $2.0-\mu \mathrm{L}$ aliquots of the mixture to pieces of Zetabind immobilization membrane (Cuno Laboratory Products, purchased from Life Science Products Inc., Denver, $\mathrm{CO}$ ) fixed to the LaserTec probe tip by double-sided adhesive tape [16]. After drying of the sample on the membrane, the probe tip was immersed in water to remove the urea. This procedure was repeated 3-10 times, depending on the final sample volumes, to concentrate analyte while removing excess urea.

All experiments were performed using $\alpha$-cyano-4hydroxycinnamic acid (Aldrich Chemical Co., Milwaukee, WI) as the matrix. Saturated matrix solutions were prepared in a 1:1 solution of acetonitrile/aqueous $0.1 \%$ TFA containing internal standards, and mixed in equal volumes with the peptide/protein sample and applied to a stainless-steel probe tip. Otherwise, matrix solution containing internal standards was applied to the probe-mounted Zetabind membrane containing the adsorbed protein sample. Matrix-protein solutions were allowed to "air-dry" and crystallize on the membrane surface prior to introduction into the mass spectrometer [16].

\section{Results and Discussion}

\section{Derivatization of Peptides with $p H M B$}

Evaluation of the new methodology and behavior of the organomercurial, $p$-hydroxymercuribenzoate ( $\mathrm{pHMB}$ ), were initially studied with somatostatin, a 14-residue peptide containing one cystine ( $M W=$ 1637.9). The MALDI mass spectrum shown in Figure 2a was recorded from a 15-pmol sample of somatostatin. No modification of the peptide was observed when mass spectral data were acquired from samples prepared in the presence of up to twenty-fivefold molar excess of $\mathrm{pHMB}$ (and in the absence of a reducing reagent), indicating no insertion of the organomercurial reagent into the disulfide bond. During incubation with a twofold molar excess of TCEP for 20 minutes at $37^{\circ} \mathrm{C}$, the cystines of similar somatostatin samples were completely reduced, as confirmed by the MALDI mass spectrum in Figure $2 b$, which shows a peak at $m / z 1640.7,[\mathrm{M}+\mathrm{H}]_{\text {calc }}^{+}=1640.9$. A similarly reduced sample of somatostatin was then incubated with a fivefold molar excess of pHMB for 5 minutes at room temperature and analyzed by MALDI directly after cocrystallization with the matrix. The MALDI mass spectrum in Figure 2c shows that the latter procedure results in derivatization of both liberated cysteine residues that constituted the disulfide bond, shifting

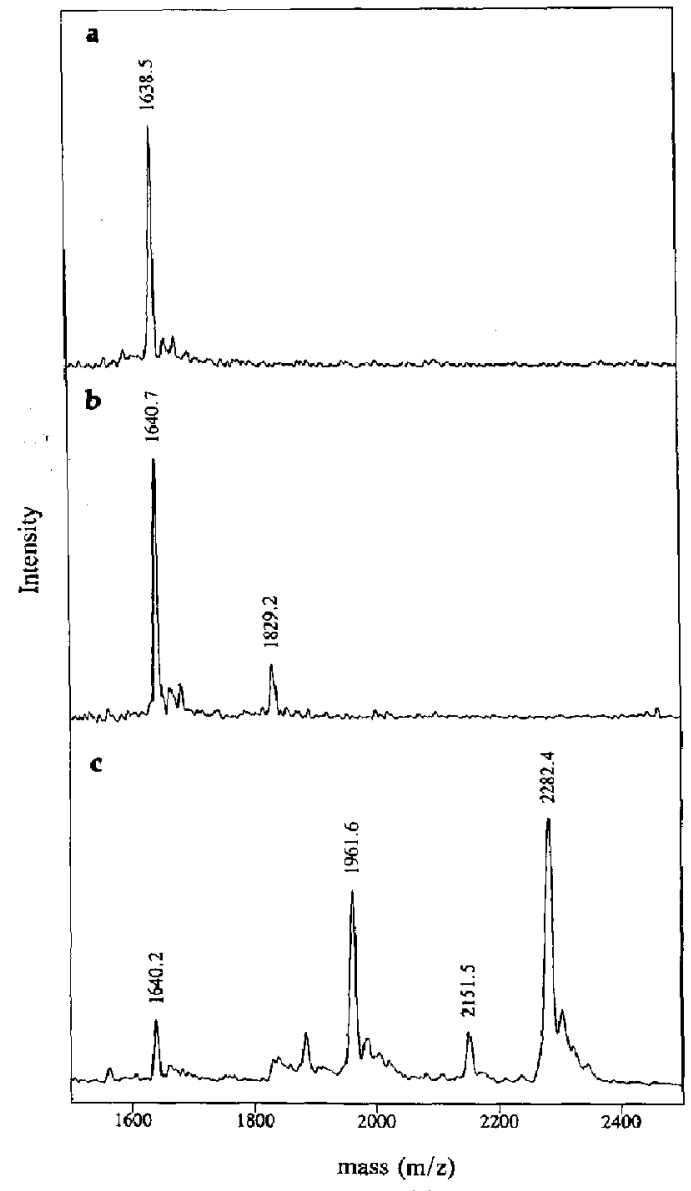

Figure 2. MALDI mass spectrum of (a) 15 pmol of somatostatin, $[\mathrm{M}+\mathrm{H}]_{\text {calc }}^{+}=1638.9$; (b) 15 pmol of somatostatin following reduction, $[\mathrm{M}+\mathrm{H}]_{\text {calc }}^{+}=1640.9$; and (c) 15 pmol of reduced somatostatin after reaction with a fivefold molar excess of pHMB over total cysteine. Peaks recorded at $m / z \quad 1961.6$ and 2282.4 represent derivatization of one and both free cysteine residues, respectively.

the mass of the analyte by $(321 \times 1) \mathrm{u}$ to $[\mathrm{M}+\mathrm{H}]_{\text {calc }}^{+}$ $=1961.9$, and by $(321 \times 2) \mathrm{u}$ to $[\mathrm{M}+\mathrm{H}]_{\text {calc }}^{+}=2282.9$. Assigruments were also made for the minor peaks in Figure $2 b$ and $c$, representing either an adduct or complex of modified somatostatin with $\alpha$-cyano-4hydroxycinnamic acid (see section on Competing Side Reactions, below).

\section{Derivatization of Proteins with $p H M B$}

Proteins may contain cysteine, cystine, or a combination of both these functionalities. To fully characterize these groups for most proteins it will be necessary to ensure that the macromolecule is sufficiently denatured to derivatize sulfhydryl groups that may not be accessible in the folded, native state ("masked cys- 
teines"). Because reduced and denatured proteins of ten tend to precipitate, solubility is typically maintained with a high concentration (2-6 M) of chaotropic reagents, such as urea or guanidine hydrochloride, in the reaction medium. However, before analysis of the sample by MALDI-MS, the denaturing agents must be removed. Standard procedures for desalting like dialysis or gel filtration cause significant protein losses when applied to low-level samples (i.e., $1 \mu \mathrm{g}$ or less); therefore, we have employed a recently developed membrane-based sample cleanup procedure (ref 16, see Experimental).

Insulin was chosen as a model compound for analysis of a protein containing only disulfide bonds. Bovine insulin (MW $=5733.5$ ) consists of three disulfide bonds, two intermolecular bonds between the insulin A-chain (reduced MW = 2339.7) and the B-chain (reduced $M W=3399.9$ ), and one intramolecular disulfide bond located within the A-chain. Treatment of native insulin with $\mathrm{pHMB}$ did not result in modification of the analyte, $[\mathrm{M}+\mathrm{H}]_{\mathrm{obs}}^{+}=5734.7$ (data not shown). Figure 3a shows the negative ion MALDI mass spectrum recorded from a sample of insulin following reduction with TCEP. The two predominant peaks in the spectrum represent the reduced $\mathrm{A}$ - and B-chains.

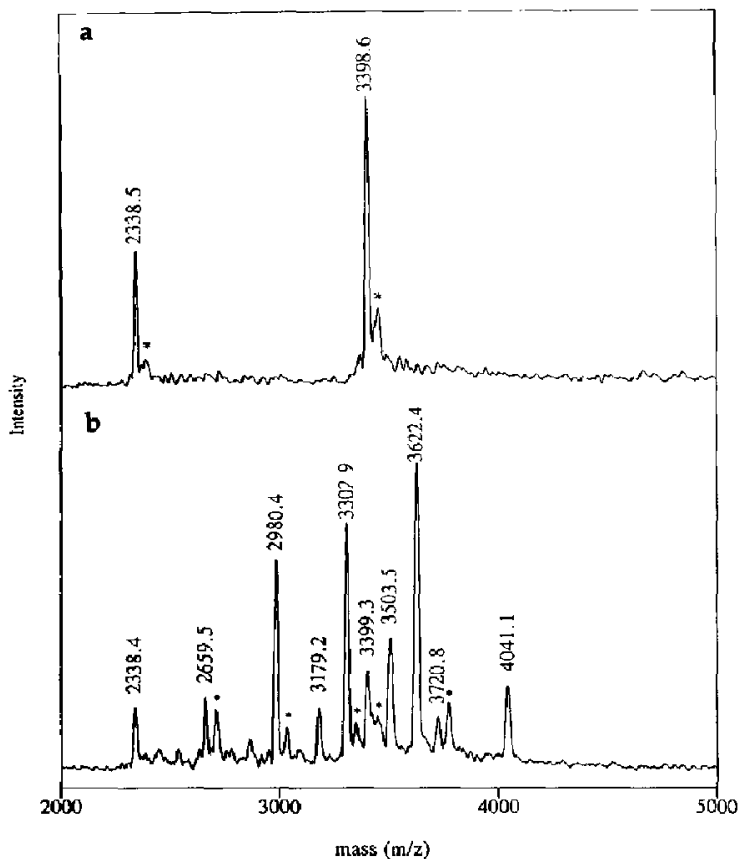

Figure 3. MALDI negative ion mass spectrum of (a) l's pmol of reduced bovine insulin displaying peaks for the A- and B-subunits; (b) 17 pmol of reduced bovine insulin after reaction with pHMB. Peaks marked with asterisks represent an oxidation of cysteine to cysteic acid ( $48 \mathrm{u}$ mass shift). See Table 1 for correlation of experimentally determined (Figure $3 \mathrm{~b}$ ) values of mass-tocharge ratios with calculated values of mass-to-charge ratios for indicated structural formulac.
In contrast, the positive ion MALDI mass spectrum (data not shown) only showed a single peak representing the reduced form of the insulin B-chain. The absence of a peak for the reduced A-chain of insulin in the positive ion MALDI mass spectrum has been reported by others [17], and may be related to the number of acidic residues present in the peptide. Reduction was nearly complete using a twofold molar excess of TCEP (over cystine) as determined by the near absence of a signal for native insulin in either the positive or negative MALDI-MS mode. In these experiments with insulin, it was necessary to process the protein in $4 \mathrm{M}$ urea because of insolubility of the reduced insulin A-chain. The MALDI mass spectrum shown in Figure $3 b$ was recorded from a reduced sample of insulin that was derivatized with $\mathrm{pHMB}$. Although the mass spectrum is complex, showing a multiplicity of peaks corresponding to partially derivatized species with quantized mass shifts of $321 \mathrm{u}$ for each $\mathrm{pHMB}$ (or $201 \mathrm{u}$ for each mercury) added, peaks shifted to $m / z 3622$ (Achain) and $m / z 4041$ (B-chain) indicate that all six cysteine residues of bovine insulin can be accounted for by $\mathrm{pHMB}$ derivatization. Table 1 lists the calculated and observed mass-to-charge ratio values for all products of the reaction between PHMB and the indicated number of SH groups. The spectrum in Figure $3 \mathrm{~b}$ indicates that some cysteines were oxidized to cysteic acid, producing a mass shift of $48 \mathrm{u}$ for every cysteic acid residue (peaks identified by asterisks). Metal ions are known to act as catalysts in the oxidation of cysteine to cysteic acid [18], therefore the PHMB may have acted as a catalyst in the oxidation process. However, some oxidized products of insulin $A$ and $B$ chain can be observed prior to treatment with pHMB (Figure 3a).

Hemoglobin A ( $\mathrm{HbA}$ ) was chosen as a model compound for thiol group analysis of a protein containing only free cysteine. Human hemoglobin A consists of an $\alpha$-chain with one free cysteine (MW $=15,126)$ and a $\beta$-chain with two free cysteines (MW $=15,867)$. The MALDI mass spectrum in Figure 4a, recorded from a 100-pmol sample of dehemed human hemoglobin A, shows peaks representing the protonated molecules for

Table 1. Calculated and observed masses for pHMBderivatized A- and B-chains of reduced bovine insulin

\begin{tabular}{lcc}
\hline Subunit chain & {$[\mathrm{M}-\mathrm{H}]_{\text {calc }}^{-}$} & {$\left[\mathrm{M}-\mathrm{H}_{\text {ots }}^{-}\right.$} \\
\hline $\mathrm{A}$ & 2338.7 & 2338.4 \\
$\mathrm{~A}+1$ pHMB & 2659.7 & 2659.5 \\
$\mathrm{~A}+2 \mathrm{pHMB}$ & 2980.7 & 2980.4 \\
$\mathrm{~A}+2 \mathrm{pHMB}+1 \mathrm{Hg}$ & 3181.3 & 3179.2 \\
$\mathrm{~A}+3 \mathrm{pHMB}$ & 3301.7 & 3302.9 \\
$\mathrm{~A}+3 \mathrm{pHMB}+1 \mathrm{Hg}$ & 3502.3 & 3503.5 \\
$\mathrm{~A}+4 \mathrm{pHMB}$ & 3622.7 & 3622.4 \\
$\mathrm{~B}$ & 3398.9 & 3399.3 \\
B +1 pHMB & 3719.9 & 3720.8 \\
B +2 pHMB & 4040.9 & 4041.1 \\
\hline
\end{tabular}




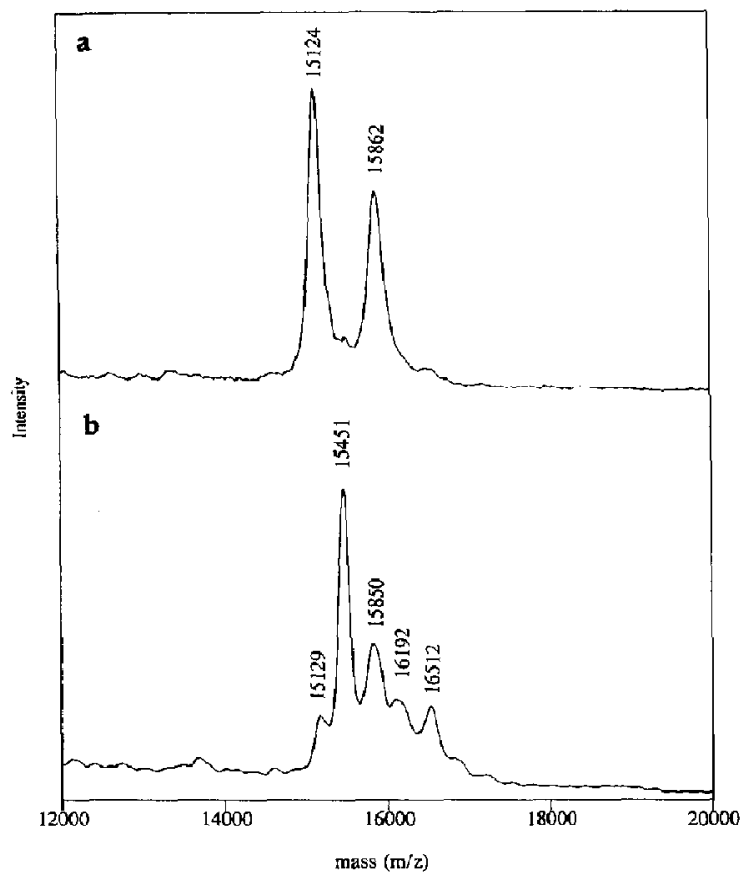

Figure 4. MALDI mass spectrum of (a) a mixture of $\alpha$ - and $\beta$-globin chains (approximately $100 \mathrm{pmol}$ ) of human hemoglobin A ( $\mathrm{HbA}),[\mathrm{M}+\mathrm{H}]_{\text {calc }}^{+}=15,127$ and 15,868 , respectively; (b) a mixture of $\alpha$ - and $\beta$-globin chains (approximately $100 \mathrm{pmol}$ ) of $\mathrm{HbA}$ following derivatization with $\mathrm{pHMB}$. The peak at $m / z$ 15,451 represents modification of the single cysteine in the $\alpha$ globin chain, while the peak at $m / z \quad 16,512$ indicates that both cysteines in the $\beta$-globin chain were derivatized.

both the $\alpha$ - and $\beta$-chains. Because the globin chains were readily soluble in $0.1 \% \mathrm{NII}_{4} \mathrm{OH}$, a urea denaturant was not used for these experiments. Figure $4 b$ shows the MALDI mass spectrum recorded from another similar aliquot of a hemoglobin A sample that had been treated with a fivefold molar excess of pHMB for 5 minutes. Assigned peaks (Figure 4b) correspond to $\mathrm{pHMB}$ modification of the single cysteine in the HbA $\alpha$-chain shifting its mass by $(321 \times 1) u$ to $[\mathrm{M}+$ $\mathrm{H}]_{\text {calc }}^{+}=15,448$, and the two cysteine residues in the $\mathrm{HbA} \beta$-chain, shifting its mass by $(321 \times 2) \mathrm{u}$ to $[\mathrm{M}+$ $\mathrm{H}]_{\text {cale }}^{+}=16,510$. Thus, the organomercurial derivatization approach can be applied to cysteine analysis in a mixture of proteins. Classical means of analyzing this sample would result in quantitation of only the total cysteine content of the mixture unless a separation was performed prior to analysis. However, assessment of the mass shifts recognized by comparing Figure $4 a$ and b correctly correlates with the known cysteine content for the mixture of human hemoglobin $\alpha$-and $\beta$-chains.

The milk protein $\beta$-lactoglobulin ( $M W=18,367$ ) was selected as a model protein containing both cysteine and cystine groups. This protein consists of five cysteine residues, four of which make up two disulfide bonds, and one of which is free. The MALDI mass spectrum of the unmodified protein (Figure $5 \mathrm{a}$ ) shows a major peak at $m / z 18,375\left([\mathrm{M}+\mathrm{H}]_{\mathrm{calc}}^{+}=18,368 \mathrm{u}\right)$, and a minor trailing shoulder at $m / z$ 18,674. The source of this shoulder is unknown, but it was found in mass spectra obtained from samples of the protein procured from two different sources (one being sequencing grade $\beta$-lactoglobulin). Because at least four different forms of $\beta$-lactoglobulin are known to exist (one of which is glycosylated), we believe that the trailing peak likely results from analysis of heteroge neous samples containing different protein forms. The MALDI mass spectrum of unreduced and pHMB-modified $\beta$-lactoglobulin (Figure $5 b$ ) shows that reaction with the organomercurial reagent shifted both peaks in Figure 5a by 321 u, suggesting that the trailing shoulder likely represents a structurally related impurity. Native $\boldsymbol{\beta}$-lactoglobulin was derivatized in the absence of urea as it was readily soluble in $0.1 \% \mathrm{NH}_{4} \mathrm{OH}$. However, reduced $\beta$-lactoglobulin required urea for solubilization. The MALDI mass spectrum shown in Figure $5 c$ was obtained from a sample of $\beta$-lactoglobulin that was denatured in $4 \mathrm{M}$ urea, reduced with a twofold molar excess of TCEP (over cystine), derivatized with a fivefold molar excess of $\mathrm{pHMB}$ (over cysteine), and desalted/concentrated on a protein transfer membrane fixed to the MALDI probe tip. Peaks that can be assigned to species resulting from derivatization of all five cysteine residues in $\beta$-lactoglobulin are observed. Even though the peak representing the fully derivatized protein is small, [M + $\mathrm{H}]_{\mathrm{obs}}^{+}$at $m / z 19,974$, its assignment is not ambiguous. The mass shift of the predominant peak in Figure 5a to $m / z 18,695$ in Figure 5b shows that a single cysteine is present in the unreduced protein. Reduction of each disulfide bond will liberate two sulfhydryl groups, which can be derivatized by pHMB. Therefore, in the spectrum of the reduced/derivatized sample (Figure 5c), the three peaks at $m / z 19,018,19,327$, and 19,640 are expected to be accompanied by a fourth peak corresponding to a fully derivatized species. The low abundance of the fully derivatized protein may have several causes. First, even after denaturation, sulfhydryl groups in large proteins may not be completely accessible to the derivatizing reagent. It is also possible that cysteine oxidation is competing with pHMB derivatization. However, we have demonstrated that complete derivatization of insulin can be accomplished with good yield even in the face of competing oxidation (Figure 3b). We are currently investigating modification of reaction conditions for larger proteins to improve the yield of the fully derivatized products.

\section{Choice of a Mercurial Reagent for Sulfhydryl Derivatization}

Although numerous types of reagents are available for derivatization of macromolecular thiols, mercury compounds were chosen for several reasons, but primarily because of the selective reactivity of mercury for 


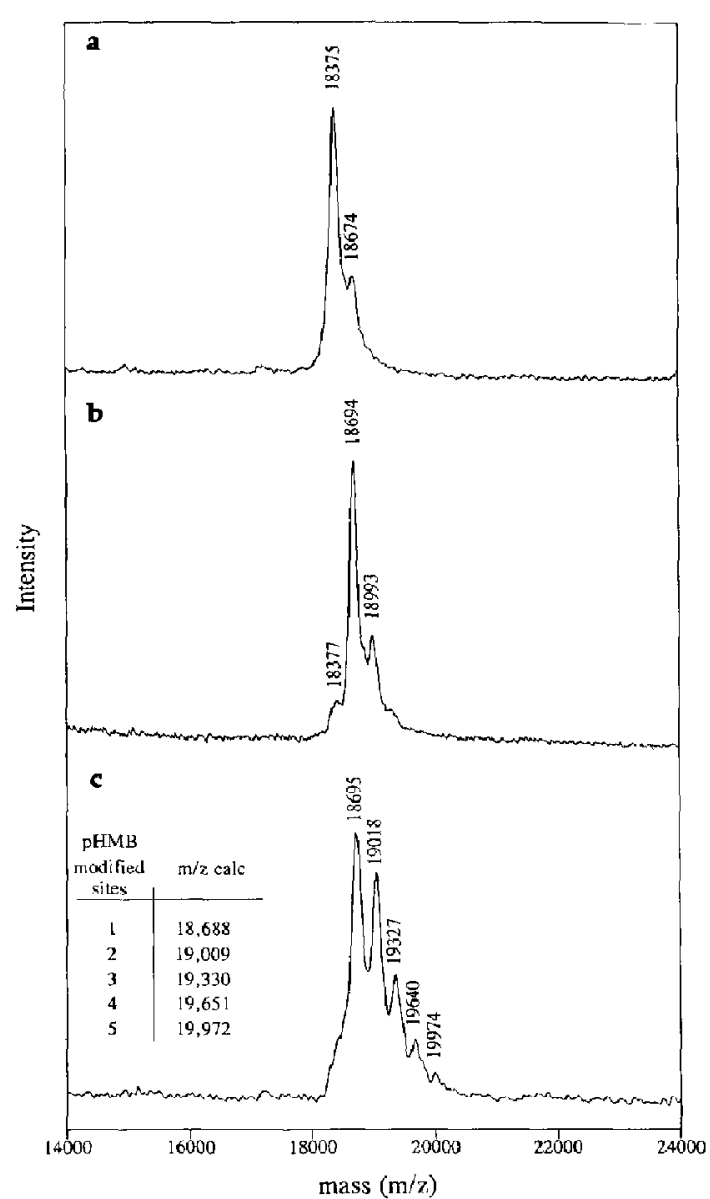

Figure 5. MALDI mass spectrum of (a) $11 \mathrm{pmol}$ of bovine $\boldsymbol{\beta}$-lactoglobulin A, $[\mathrm{M}+\mathrm{H}]_{\text {calc }}^{\mathrm{r}}=18,368$; (b) 11 pmol of $\beta$-lactoglobulin A following derivatization of the single free cysteine with $\mathrm{pHMB}$ under nonreducing conditions $[\mathrm{M}+\mathrm{H}]_{\text {calc }}^{+}=18,688$; (c) $11 \mathrm{pmol}$ of denatured and reduced $\beta$-lactoglobulin $A$ following derivatization with pHMB. Peaks in c represent modified analngs of the reduced analyte with progressively increasing numbers of $\mathrm{pHMB}$ with the available free-SH groups listed in the insert.

sulfhydryl groups. Sufficient selectivity is not always achieved with many types of alkylating reagents such as the maleimides, which have been found to react with the imidazole group of histidine and the $\alpha$-amino group of amino acids [19]. Similarly, the haloacids and their amides, also popular reagents for sulfhydryl derivatization, have been found to react with the thioether group of methionine [20]. On the other hand, mercury compounds are highly specific reagents for the derivatization of sulfhydryl groups. The selectivity is related to the strong mercury-sulfur interaction; the dissociation constants for the mercaptide formed between mercuric ion $\left(\mathrm{Hg}^{2+}\right)$ and cysteine or methyl mercury $\left(\mathrm{CH}_{3} \mathrm{Hg}^{+}\right)$and cysteine are $10^{-15.8}$ and $10^{-20.3}$, respectively [21]. Although mercuric ions show the strongest binding to sulfhydryl groups, they are not suitable derivatizing reagents because divalent mercury will form mercaptides of the type (RS) ${ }_{2} \mathrm{Hg}$ [13], which will produce anomalous mass spectral results. Monovalent organomercurials, however, are only capable of forming mercaptides of the type (RS) $\mathrm{HgR}$, and will only cleave disulfide bonds and react with the liberated sulfhydryl groups under harsh conditions (i.e., high concentration of the organomercurial, elevated temperatures, and prolonged incubation times) [22].

Another reason for our choice of organomercurial reagents is that these compounds promote large mass shifts in the mass spectrum of the derivatized protein. This is important in the analysis of large proteins by MALDI-TOF-MS where mass resolution is limited. In this report we have primarily investigated p-HMB and $\mathrm{p}$-HMPS as derivatizing reagents that promote mass shifts of $321 \mathrm{u}$ and $357 \mathrm{u}$, respectively. Although pHMPS and $\mathrm{pHMB}$ produced similar results in classifying sulfhydryl groups as being either cysteine or half-cystine, only data obtained from $\mathrm{pHMB}$ are presented here because of its convenient purification. MALDI-MS data obtained from peptides and proteins derivatized with pHMPS contained significantly more peaks, representing modification of the macromolecule by mercuric ion (201 u mass shift). We also have derivatized sulfhydryl groups with organomercurials such as merbromin ( $\Gamma$ igure $1 \mathrm{a}$ ), which produces a mass shift of $690 \mathrm{u}$. While $\mathrm{CH}_{3} \mathrm{Hg}^{+}$has been used in amperometric titrations [15], it was not used in this work because it was not readily available.

Many of the organomercurial compounds considered for this application were contaminated by mercuric ion, which produced additiond peaks in the MALDI mass spectra. This contamination may arise from photodegradation as many organomercurial compounds are light-sensitive. Even though the mercuric ion contamination may be very small, the high affinity of mercuric ion for sulfhydryl groups (over that of monovalent organomercurials) will produce significant mercuric ion derivatization. Purification of the organomercurial compound prior to its use is therefore essential for desirable derivatization of the peptide or protein. Although merbromin and pHMPS (Figure 1a) are readily soluble in either acidic or basic aqueous solutions, pHMB is only soluble in basic solutions ( 2.8 $\mathrm{mg} / \mathrm{mL}$ at a $\mathrm{pH}$ above 10.0 ). We made use of the relative insolubility of $\mathrm{pHMB}$ in acidic conditions to purify it by washing with $0.1 \%$ TFA $(\mathrm{pH}=2.2)$ prior to its use. This treatment conveniently removes most of the mercuric ion contamination. Because of its limited solubility at low or neutral $\mathrm{pH}, \mathrm{pHMB}$ derivatization requires all reactions to be performed under basic conditions. Therefore, reactions were conducted in solutions containing $0.1 \% \mathrm{NH}_{4} \mathrm{OH}(\mathrm{pH}=10.8)$. Disulfide bonds can be cleaved in alkaline media [23], but we found that cleavage will not occur if derivatization reactions are performed in less than 30 minutes. 
We found that derivatization of peptides and proteins occurred in less than 5 minutes using basic conditions. Reagents such as pHMB or pHMPS are expected to be less reactive under basic conditions in which association of hydroxide ion and the mercury substituent of the organomercurial is favored [22]. However, because the reactive form of the sulfhydryl group is the ionized thiolate, performing the reaction under acidic conditions would have the adverse effect of limiting the reactivity of the sulfhydryl group.

\section{Choice of Reducing Reagent}

Common reducing reagents for proteins such as mercaptoethanol or dithiothreitol cannot be used in the derivatization procedures described in this report because they will react with the organomercurial reagent. Our preliminary experiments indicated that $100 \mathrm{mM}$ sodium borohydride was an effective reducing reagent, but if excess reagent was not completely destroyed and removed, it interfered with the matrix-analyte crystallization process and prevented the acquisition of useful MALDI spectra. Also $r$ we found that in some experiments utilizing sodium borohydride reduction, spurious MALDI-MS signals were recorded. This was likely due to peptide bond cleavage by the sodium borohydride, as has been reported by others [24]. Tertiary phosphines are known to be powerful reducing reagents that cause no modification of residues other than cystine and are effective in stoichiometric ratios [25]. Although many tertiary phosphines are insoluble in aqueous media and have to be used as suspensions, we chose TCEP because of its water solubility [26]. TCEP is an effective reducing reagent under basic conditions, requires no more than a twofold molar excess (over cystine) for complete reduction of disulfide bonds, and does not interfere with the matrix-analyte crystallization process prior to analysis by MALDI-MS.

\section{Competing Side Reactions}

Because thiols also can add across polarized or easily polarizable double bonds [27], reaction with matrices, especially the cinnamic acid derivatives, would be anticipated. This type of competing reaction can be seen in the MALDI mass spectrum obtained from a reduced sample of somatostatin analyzed by using $\alpha$-cyano-4-hydroxycinnamic acid as the matrix (Figure 6a). After 12 hours of exposure of somatustatin to matrix (solid phase) on a stainless-steel probe tip, data were recorded showing extensive reaction of both cysteine residues with tx-cyano-4-hydroxycinnamic acid. The abundance of the matrix-thiol derivative observed in Figure 6a, $[\mathrm{M}+\mathrm{H}]_{\mathrm{obs}}^{+}=1829.6\left([\mathrm{M}+\mathrm{H}]_{\text {calc }}^{+}=\right.$ 1830.8 ), is nearly the same as that of the protonated molecule of somatostatin. In contrast, matrix-adduct ions typically have only about $5 \%$ (or less) of the abundance of the protonated analyte in the typical
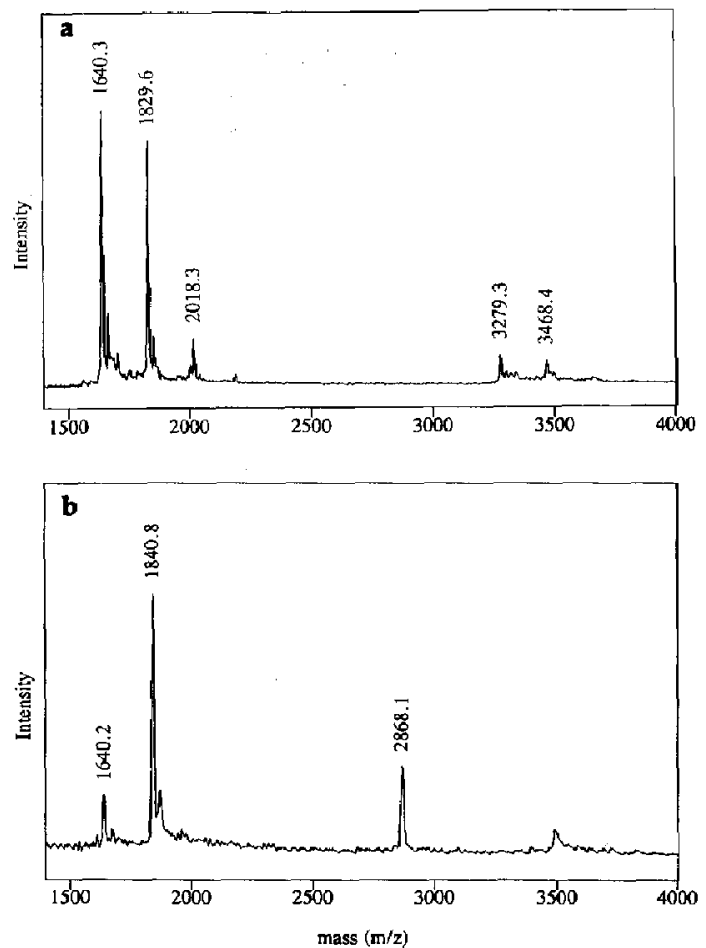

Figure 6. MALDI mass spectrum of (a) reduced somatostatin (15 pmol) that was exposed to $\alpha$-cyano-4-hydroxycinnamic acid matrix for 12 hours prior to obtaining the MALDI mass spectrum. Peaks recorded at $m / z \quad 1829.6$ and 2018.3 represent modification of the peptide with one and two $\alpha-c y a n o-4$-hydroxycinnamic acid moieties, and the peaks recorded at $m / z 3279.3$ and 3468.4 represent a disulfide exchange reaction that occurred between two somatostatin peptides and modification of the two somatostatir peptides by one $\alpha$-cyano-4-hydroxycinnamic acid moiety. Spectrum b was obtained from an aliquot (15 pmol) of the somatostatin sample used to record the mass spectrum shown in Figure 2c, but after remaining in the $\mathrm{pHMB}$ reaction mixture for 24 hours, showing modification of the reduced peptide with mercuric ion. The peak at $m / z 2868.1$ represents doubly charged insulin.

MALDI mass spectrum. Other peaks assigned in Figure 6 a represent somatostatin modified by two $\alpha$ cyano-4-hydroxycinnamic acid groups, a dimer of somatostatin (disulfide exchange product), and a dimer of the somatostatin matrix complex (see legend to Figure 6).

We have found that matrix-cysteine reaction products are formed in the solid phase, in a time-dependent manner. However, if cysteine-containing peptides are analyzed immediately after sample preparation, much less reaction of the analyte with $\alpha$-cyano-4-hydroxycinnamic acid is observed. The $\mathrm{pH}$ of the derivatizing solution is also important in the modification of cysteinyl peptides / proteins by matrices such as $\alpha$-cyano4-hydroxycinnamic. Adjusting the $\mathrm{pH}$ of the solution containing the analyte below $\mathrm{pH}=3.0$ maintains the thiol groups in protonated form, and thus minimizes the formation of matrix-thiol addition products. Alter- 
natively, "matrix derivatization" of sulfhydryl-containing analytes can be avoided by using a matrix less reactive towards sulfhydryl groups (e.g., 2,5-dihydroxybenzoic acid) or by driving the organomercurialsulfhydryl reaction to completion before adding matrix. The latter solution, however, will not always be effective because a large excess of organomercurial reagent is required, which also may promote adverse side reactions such as the reduction of disulfide bonds [22].

Another competing reaction sometimes observed was the modification of cysteine with mercuric ion or other mercury contaminants that may be present in the derivatizing reagent. Although the pHMB used in all experiments was washed prior to its use, some contaminating mercuric ion remained. As was the case in minimizing matrix-thiol derivative formation, we also have observed that modification of analyte sulfhydryl groups by contaminating mercuric ion can be minimized by analyzing samples immediately after derivatization and matrix cocrystallization. The consequence of prolonged derivatization reaction times can be seen in Figure 6b, which is the MALDI mass spectrum obtained from an aliquot of the same sample used to obtain the data in Figure $2 c$, but after remaining in the reaction mixture for 24 hours. Only peaks corresponding to reduced somatostatin, and somatostatin, modified by one mercuric ion can be seen. The data in Figure 6 show that with time mercuric ion can displace the organomercurial reagent, even though the concentration of mercuric ions may be very low. This result is expected, given the much higher association constants of mercuric ion for cysteine over that of an organomercurial compound [21]. Derivatization of a peptide with merbromin was successful in promoting a mass shift of the analyte by $690 \mathrm{u}$; however, many other unassigned signals were observed, indicating the need for purification of such compounds prior to their use as derivatizing reagents.

\section{Conclusions}

Challenges in successfully applying chemical derivatization to protein samples to obtain structural information by mass spectrometric analyses include: finding selective reactions that will avoid ambiguous results, using reagents and conditions that do not result in secondary modifications of any amino acids, and developing protocols that will allow for the removal of excess/interfering reagents when working on small amounts of sample. In this report the use of microscale organomercurial derivatization of protein thiols, combined with sample adsorption onto transfer membranes to facilitate excess reagent removal, has been successfully applied in the analysis of protein cysteine/cystine content. Reaction of sulfhydryl groups with organomercurial reagents produces large mass shifts in the MALDI-TOF spectrum, facilitating recog- nition of modified analyte molecules, despite the inherent limitations in mass resolution of this technique. Appropriate treatment of the protein with denaturing and reducing agents prior to derivatization by organomercurials and subsequent analysis by MALDIMS can provide data related to the number of free sulfhydryls and disulfide bonds present. Experiments performed in the presence of a denaturant will provide information about free and masked cysteines. Our studies have demonstrated that reagent purification and expeditious analysis of the sample after derivatization minimizes potential side reactions that could complicate spectral interpretation. Continuing work in this area will be directed toward improving the reaction efficiency and extending the methodology to submicrogram samples.

\section{References}

1. McMurray, C. H.; Trenton, D. R. Biochem. J. 1969, 115, 913-921.

2. Boyer, P. D. J. Am. Chem. Soc. 1954, 76, 4331-4337.

3. Alexander, N. M. Anal. Chem. 1958, 30, 1292-1294.

4. Kirley, T. L. Anal. Biochem. 1989, 180, 231-236.

5. Moore, J. E. I. Biol. Chem. 1963, 238, 235-237.

6. Crestfield, A. M.; Moore, S.; Stein, W. H. J. Biol. Chem. 1963, $238,622-627$

7. Weil, L; Seibles, T. S. Arch. Biochem. Biophys. 1961, 95, $470-473$

8. Scoffone, E.; Fontana, A. In Protein Sequence Deternination; Needleman, S. B., Ed.; Springer-Verlag: New York, 1975; p 190.

9. Simpson, R. J.; Neuberger, M. R.; Liu, T.-Y. J. Biol. Chem. 1963, 251(7), 1936-1940.

10. Yip, T.-T.; Hutchens, T. W. FEBS 1992, 308(2), 149-153.

11. Caprioli, R. M.; Whaley, B. In Techniques in Protein Chemistry II; Academic Press: San Ijiego, 1991; pp 48, 497-510.

12. Torchinsky, Y. M. In Sulfur in Proteins; Metzler, D., Translation Ed; Pergamon: Oxford, 1981; p 41.

13. Jovin, T. M.; England, P. T.; Kornberg, A. I. Biol. Chem. 1969, 244, 3009-3018.

14. Chianconc, E.; Currell, D. L.; Vecchini, P.; Antonini, E.; Wyman, J. I. Biol. Chem, 1970, 245(16), 4105-4111.

15. Leach, S. J. Aust. J. Chem. 1960, 13, 520-529.

16. Zaluzec, E. J.; Gage, D. A.; Allison, J.; Watson, J. T. I. Am. Soc. Mass Spectrom. 1994, 5, 230-237.

17. Fisher, W. H.; Rivier, J. E.; Craig, A. G. Rapid Comun. Mass Spectrom. 1993, 7, 225-228.

18. Friedman, M. In The Chemistry and Biochemistry of the Sulfhydryl Group in Anino Acids, Peptides and Proteins; Pergamon: Oxford, 1973; pp 25-72.

19. Brewer, C. F.; Riehm, J. P. Anal. Biochem. 1967, 18, 248-255.

20. Goren, H. J.; Barnard, E. A. Biochemistry 1970, 9(4), 959-973.

21. Simpson, R. B. T. Am. Chem. Soc. 1961, 83, 4711-4717.

22. Torchinsky, Y. M. In Sulfur in Proteins; Metzler, D., Translation Ed.; Pergamon: Oxford, 1981; p 75.

23. Donovan, J. W.; White, T. M. Biochemistry 1971, 10, 32-38.

24. Andersson, I. O. Arch. Biochem. and Biophys. 1969, 133, 277.

25. Rüegg, U. T.; Rudinger, J. Methods Enzymol. 1977, 47, 111-116.

26. Burns, J. A.; Butler, J. C.; Moran. J.; Whitesides, G. M. J. Org. Chem. 1990, 56, 2648-2650.

27. Torchinsky, Y. M. In Sulfur in Proteins; Metzler, D., Translation Ed.; Pergamon: Oxford, 1981; pp 18-25. 